\title{
A Comprehensive Review of Evidence-Based Strategies to Prevent and Treat Postoperative lleus
}

\author{
Sara K. Story ${ }^{\mathrm{a}}$ Ronald S. Chamberlain ${ }^{\mathrm{b}}$ \\ aSaint George's University School of Medicine, Grenada, West Indies; 'b Surgery, Saint Barnabas Medical Center, \\ Livingston, N.J., and Surgery, University of Medicine and Dentistry of New Jersey, Newark, N.J., USA
}

\section{Key Words}

Alvimopan • Bowel distention • Methylnaltrexone •

Postoperative ileus

\begin{abstract}
Background: Postoperative ileus (POI) is a common complication of abdominal and several other surgeries leading to increased hospital stay and healthcare costs. POI also contributes towards numerous postsurgical comorbidities including deep vein thrombosis and pneumonia. $\mathrm{POI}$ is characterized by bowel distention and lack of bowel sounds, flatus and bowel movements. The causative mechanism is not fully understood and may be multifactorial including disorganized electrical activity, activation of inflammatory mediators and the use of opioid analgesics. Methods: A selective review of the literature pertaining to the prevention and treatment of adynamic ileus and POI was completed. More specifically we sought to evaluate RCTs, meta-analyses, consensus statements and articles providing graded evidence-based data on POI prevention and treatment. $\boldsymbol{R e}$ sults: Perioperative strategies employed to prevent or limit the duration of $\mathrm{POI}$ include avoidance of preoperative fasting and mechanical bowel preparation, use of epidural-local anesthetics, implementation of minimally-invasive surgical techniques, and modification of pain management strate-
\end{abstract}

gies to limit opioid administration among others. Conclusion: Though many of these strategies have proven beneficial, no single approach has demonstrated the ability to prevent or treat POI. However, when these strategies are used in combination as part of a fast-track multimodal treatment plan, there is a significant decrease in time to return of normal bowel function and a shortened hospital stay. Additional studies are needed to make specific recommendations regarding which components of fast-track protocols are most beneficial.

Copyright $\odot 2009$ S. Karger AG, Basel

\section{Introduction}

Postoperative ileus (POI) is the most common cause of prolonged hospital stay following abdominal surgery [1]. A recent analysis of 161,000 major bowel resections performed between 1999 and 2000, as listed in the Health Care Financing Administration (HCFA) database, found that the length of stay increased by 5 days in cases with a coded POI [2]. This same analysis found the readmission rate for patients with documented POI to be $3.6 \%$ compared to $0.2 \%$ in all other patients $[1,2]$. In these cases the extra hospitalization costs attributed to POI was USD 1.14 billion, and total healthcare cost was significantly

\section{KARGER}

Fax +4161306 1234 E-Mail karger@karger.ch www.karger.com

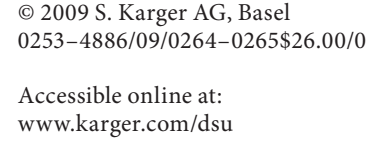

Ronald Scott Chamberlain, MD

94 Old Short Hills Road

Livingston, NJ 07039 (USA)

Tel. +1 973322 5195, Fax +1 9733222471

E-Mail rchamberlain@sbhcs.com 
higher (USD 16,000/patient with POI vs. USD 10,000/patient without POI; $\mathrm{p}<0.01$ ) [1]. An alternate study by Iyer and Saunders [3] had similar findings when looking at 17,896 patients listed in the Perspective Comparative $\mathrm{Da}$ tabase (PCD) who had undergone partial excision of the large intestine. Of this cohort, $17.4 \%$ (3115) had a secondary diagnosis of POI and a significantly longer average length of stay $(13.75 \pm 13.33$ days with POI vs. $8.85 \pm$ 9.49 days without POI; $\mathrm{p}<0.001)$.

POI contributes to many comorbid postsurgical complications which include delayed surgical wound healing and ambulation, atelectasis, pneumonia and DVTs [4]. In addition to contributing towards postoperative morbidities, these complications also increase the length of hospital stay, resource use and associated healthcare costs [4]. For example, the relationship between POI and deep vein thrombosis (DVT) was examined by reviewing the clinical course of 2,949 patients undergoing 3,364 consecutive primary and revision total hip and total knee arthroplasties, radical debridements, and reimplantations. Ileus occurred in 62 patients $(2.1 \%)$ and symptomatic DVT in $51(1.7 \%)$. In patients with ileus, the incidence of DVT was $8.1 \%(\mathrm{OR}=5.5 ; \mathrm{p}=0.0036)$. Symptomatic pulmonary embolism occurred in 7 patients who did not have ileus $(0.24 \%)$; in patients with ileus, the incidence was $3.2 \%(\mathrm{OR}=19.6 ; \mathrm{p}<0.0082)$ [5]. In addition, $\mathrm{POI}$ is associated with a significantly higher in-hospital mortality compared with patients not experiencing POI (mortality: $6.5 \%$ vs. $2.3 \%, \mathrm{p}<0.01$ ) [1]. The fiscal benefit which can accrue from decreasing hospital stay by even a single day is substantial and warrants close scrutiny into the mechanisms and prevention of POI [3]. In this review, we examine the causes, prevention and treatment of POI and discuss current evidence-based strategies that may limit associated morbidity and mortality as well as decrease length of stay and associated hospital costs.

\section{Methods}

A systematic review of the English language scientific literature from 1978 to 2008 was performed using the Medline, the Embase, and the Cochrane Central Register of Controlled Trials to obtain access to all publications including randomized controlled trials (RCTs), meta-analyses and systemic reviews pertaining to the causes, prevention and treatment of POI. The search strategy described by Robinson and Dickersin [6] was followed using key words such as ileus, bowel obstruction, prokinetic, mechanical, adynamic, treatment, and prevention. Articles were retrieved and reviewed, and the evidence from medical literature in combination with consensual clinical experience was applied to develop strategies to prevent and treat POI.

\section{Definition of Postoperative lleus}

POI is described as a transient impairment of bowel motility that may occur after major surgery $[7,8]$. An initial delay of bowel motility for 1-2 days appears to be an obligatory part of the normal surgical recovery process; however, prolongation of this period has been associated with the development of substantial in-hospital morbidity and mortality $[8,9]$. POI is considered an iatrogenic condition occurring with highest frequency and duration in association with abdominal surgeries, though it is also a consequence of other surgical procedures, including hip-fracture repair, spine procedures, neurosurgical procedures, arthroscopic surgery and others $[5,10,11]$.

The underlying pathology of POI is best described as a lack of coordinated bowel activity. Postoperative hypomotility affects all segments of the gastrointestinal (GI) tract and recovery differs by segment [12-14]. Inhibited motility in the small intestine is usually transient, recovering within several hours of surgery $[12,14,15]$. Gastric motility typically recovers within $24-48 \mathrm{~h}$ after surgery $[5,9]$, whereas the colon, the final portion of the GI tract to return to normal, usually recovers $48-72 \mathrm{~h}$ postsurgery [12-16]. Recovery of colonic motility is usually the limiting factor in resolving POI $[15,17]$.

\section{POI: Who Is at Risk?}

Compelling data which permit the accurate prediction of patients most at risk for POI are lacking; however, several studies have examined this issue. In a study of 666 patients with nonruptured abdominal aortic aneurysms, logistic regression analysis showed POI to be related to aortoiliac occlusive disease, deterioration of renal function, prolonged ventilation, and preoperative history of angina [18]. A recent retrospective study of 88 abdominal surgery patients found that the duration of POI correlates with total surgery time, blood loss and total opiate dose [19]. Gervaz et al. [20] studied 124 patients undergoing laparotomy for colectomy (median age, 68 years) and found the duration of ileus significantly reduced in those cases performed by a colorectal surgeon and when opioid analgesia lasted for less than 2 days.

Mechanical trauma of the GI tissue and the release of inflammatory mediators and cytokines have been implicated in the development of POI [8]. Given that fact, it might be expected that those undergoing a minimally invasive or laparoscopic procedure would have less risk of developing POI than those undergoing an open proce- 
dure [19]. Holte et al. [21] reviewed 4 studies focusing on the effects of laparoscopic surgery on POI and found varied results with two studies showing that laparoscopic procedures reduced POI and 2 studies showing no difference.

Though POI is most often associated with abdominal surgery, it is also seen in cardiothoracic surgery, hip fracture repair, spine procedures, neurosurgical procedures, arthroscopic surgery, gynecologic surgery, genitourinary surgery, and abdominal wall herniography among others $[5,10,11]$. Notably, POI is a complication of up to $4 \%$ of both total hip and total knee arthroplasties and abdominal hysterectomies $[1,5]$.

\section{POI: Clinical Spectrum of Presentation}

Gastrointestinal motility is expected to return to normal within 2-3 days postsurgery; when this does not occur, POI should be suspected [12-16]. That said, diagnosing POI can be complicated as the presentation is variable and difficult to distinguish from a mechanical bowel obstruction. In both cases, abdominal X-rays typically show small bowel distention with air fluid levels at various points in the intestine. Although the shape of the air fluid levels (J loops or U loops) may be suggestive of mechanical small bowel obstruction over POI, neither these findings nor evidence of air in the colon is diagnostic since the latter can be seen with partial small bowel obstruction as well.

Clinically, POI is characterized by bowel distension and lack of bowel sounds, flatus and bowel movements [7]. Symptoms include nausea, vomiting and stomach cramps [12]. Other potentially adverse effects of POI include increased postoperative pain; delayed oral intake; poor wound healing; delayed postoperative mobilization; increased risk of pulmonary complications, including pneumonia, pulmonary embolism, and atelectasis; prolonged hospitalization; decreased patient satisfaction and increased health care costs [15].

\section{POI: Mechanism of Disease}

After surgery, inhibited motility of the GI tract is universally related to disorganized electrical activity and lack of coordinated propulsion $[15,22]$. The mechanisms involved in POI and the disorganized electrical activity following surgery are not well defined and are likely multifactorial. More specifically, a variety of factors worth addressing are inhibitory sympathetic input; release of hormones, neurotransmitters and inflammatory mediators including cytokines, prostaglandins and nitric oxide $[7,12,15]$. Opioids, which are used universally as analgesics following various surgeries, also have a major importance in the pathogenesis of POI due to the depressive effects on GI transit [21]. Excessive intravascular volume given in the perioperative period may also contribute to POI development by causing intestinal edema. Figure 1 provides a complete outline of the most significant mechanisms associated with the development of POI.

\section{POI: Prevention and Treatment Strategies}

A number of perioperative strategies have been instituted or modified attempting to prevent the development or reduce the incidence and/or duration of POI (table 1). Some of these have shown independent benefits, whereas others might be beneficial only in the context of a multimodality approach. Still others incorporated in the past have been shown to be counterproductive, and their use is now discouraged based on more recent information.

\section{Preoperative Bowel Preparation}

Perioperative mechanical bowel preparation has been a cornerstone of colon surgery for decades; however, recent studies suggest it may be unnecessary and even deleterious [23]. Bucher et al. [24] reported that mechanical bowel preparation can lead to increased risk of anastomotic leaks and does not decrease the risk of septic complication. A systemic review of 9 RCTs including over 1,500 patients by Guenaga et al. [25] also found that mechanical bowel preparation did not decrease the incidence of anastomotic leaks, but actually increased the rate at which they occur as well as increasing the rate of wound infections. A randomized prospective trial of patients undergoing elective colorectal surgery demonstrated similar results, finding no benefit to routine bowel preparation [23]. Finally, Shafii et al. [26] evaluated 86 patients undergoing cystectomy and urinary diversion and reported that bowel preparation significantly increased incidence of POI and length of hospital stay.

\section{Probiotics and Carbohydrate Loading}

In recent years, new information promoting the administration of preoperative nutritional support to assist in bowel recovery following surgical procedures has begun to emerge. Probiotics are the most investigated nutritional aid to date. A 2006 review by Bengmark and Gil 


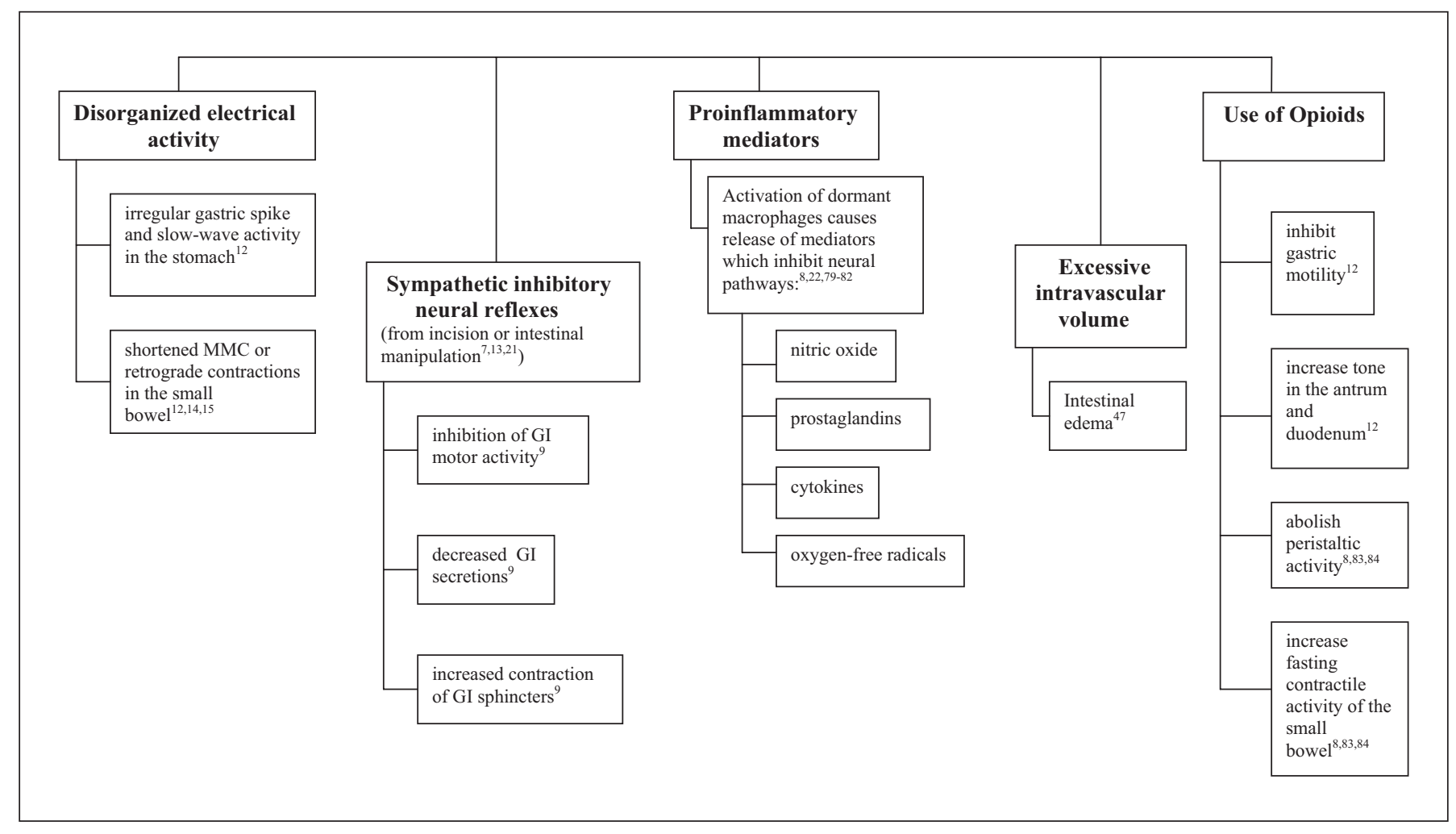

Fig. 1. Mechanisms of POI.

Table 1. Therapeutic modalities for the prevention and treatment of POI

\begin{tabular}{lll}
\hline Treatment modality & Effect on POI & Level of evidence* \\
\hline $\begin{array}{l}\text { Nonpharmacological methods } \\
\text { Nasogastric decompression }\end{array}$ & $\begin{array}{l}\text { no demonstrable benefit shown } \\
\text { increased overall complications } \\
\text { probably beneficial }\end{array}$ & Ia \\
Minimally invasive surgery & no demonstrable benefit shown & Ia \\
Early ambulation & Ib \\
Early enteral feeding & possibly beneficial & Ia \\
Gum chewing ('sham-feeding') & & Ia \\
\hline $\begin{array}{l}\text { Pharmacological methods } \\
\text { Stop routine preoperative bowel preparation }\end{array}$ & beneficial & \\
Limited intravenous fluids administration & probably beneficial & Ia \\
Epidural analgesia & beneficial & Ib \\
Preoperative probiotics administration & possibly beneficial & Ia \\
Preoperative carbohydrate loading & probably beneficial & II \\
Preoperative COX-2 inhibitors & probably beneficial & probably beneficial \\
Postoperative administration of opioid antagonists & may be beneficial & II \\
Prokinetic agents & beneficial & Ib \\
Multimodal fast-track approaches & Ia \\
\hline
\end{tabular}

* Levels of evidence categories taken from the World Health Organization http://www.euro.who.int. 
[27] found that using oral preparations of specific lactobacillus in the pre- and postoperative period may help maintain GI motility and prevent POI. However, preoperative use of these preparations has not been widely adopted and further studies are still to determine the significance of their use.

Traditionally, preoperative orders for patients have included fasting overnight before surgery to avoid complications during surgical procedures. More recently, studies have shown that using carbohydrate-rich liquids within hours prior to surgery shortens bowel recovery time postoperatively [28-30]. Noblett et al. [28] compared 36 patients of which one third were given a carbohydrate drink prior to surgery. They found that this group had a significant decrease in hospital stay when compared with those that fasted or were given an equivalent amount of water prior to surgery. A trend towards earlier normalization of GI function in the carbohydrate group was found, although it was not significant [31]. These findings were mirrored in literature reviews performed by Fearon and Luff [30], as well as Nygren et al. [29].

\section{Epidural Local Anesthetics}

As outlined earlier (fig. 1), several inhibitory reflexes in the GI tract have been proposed to play a role in POI, including activation of inhibitory reflexes originating from the incision and/or manipulation of the intestines $[7,21]$. It has been hypothesized that epidural local anesthetics may decrease POI by blocking these afferent sympathetic inhibitory reflexes, as well as efferent sympathetics with a concomitant increase in splanchnic blood flow. Systemic absorption of local anesthetics may also have anti-inflammatory effects [7, 32, 33].

Randomized clinical trials examining continuous thoracic epidural blockade with local anesthetics for more than $24 \mathrm{~h}$ have reported a decrease in POI compared with systemic opioid administration [21, 34]. Furthermore, a meta-analysis of 5 randomized clinical trials with 261 patients concluded that epidural local anesthetics reduced POI (measured as time to the first passage of stool) by $37 \mathrm{~h}$ compared with systemic opioids and $24 \mathrm{~h}$ compared with epidural opioids [34]. However, despite this positive effect on POI, epidural local anesthetics alone have not been shown to decrease hospital stay [35, 36]. A recent meta-analysis of 16 trials found that whereas epidural anesthesia significantly decreased the duration of POI compared with parenteral opioid analgesia after colorectal surgery (weighted mean difference (WMD) -1.55 days; $95 \%$ CI -2.27 to -0.84 ), it did not influence the duration of hospital stay (WMD 0.07 days;
$95 \%$ CI -0.40 to 0.54 ) [36]. However, when epidural anesthetics were incorporated as part of a multicomponent fast-track perioperative care program, Wind et al. [37] demonstrated shorter hospital stays after elective colorectal surgery.

\section{COX-2 Inhibitors}

Because of the role of prostaglandins in the inflammatory response, it has been suggested that NSAIDs or COX2 inhibitors might be used to increase GI motility as well as to reduce opioid use postoperatively [21]. Perioperative administration of COX-2 inhibitors has been found to reduce opioid administration, pain and vomiting after joint replacement surgery [38-40]. Rofecoxib, administered perioperatively, was found to be an effective component of multimodal analgesia for controlling postoperative pain after total knee arthroplasty [38]. Likewise, valdecoxib in combination with morphine was found to provide multimodal analgesia that reduced both pain and opioid use, and increased patient satisfaction following knee replacement surgery [40]. Administration of parecoxib sodium with PCA morphine resulted in significantly improved postoperative analgesic management after total hip arthroplasty, as defined by reduced opioid requirement, lower pain scores, reduced time on PCA morphine and higher global evaluation ratings [39]. Although similar studies with COX-2 inhibitors used in patients undergoing abdominal surgery are limited, a recent study with 40 patients found evidence that COX-2 inhibitors had opioid-sparing effects and reduced ileus after colorectal resection [31]. In patients administered valdecoxib $40 \mathrm{mg}$ pre- and postoperatively, bowel sound and first bowel movement appeared at medians of 12 and $72 \mathrm{~h}$ versus 24 and $84 \mathrm{~h}$, respectively, in controls ( $\mathrm{p}<$ 0.05 ). Tolerance of solid diet was at a median of $60 \mathrm{~h}$ with discharge at a median of 4 days when patients received valdecoxib versus $72 \mathrm{~h}$ and 6 days in controls $(\mathrm{p}<0.05$ and $\mathrm{p}<0.01$, respectively) [31]. It is noteworthy to mention that cardiovascular complications potentially attributable to COX-2 inhibitors have significantly limited their current use and study.

\section{Laparoscopic Surgery}

Because of experimental evidence linking mechanical trauma of GI tissue to inflammatory factors and cytokines involved in POI [8], one might expect decreased POI rates following laparoscopic as opposed to open surgical procedures. However, a review of 4 studies that examined the effects of laparoscopic surgery on POI found mixed results [21]. In 2 studies, laparoscopic surgery re- 
duced POI, whereas in the other 2 studies it did not [21, 41-44]. Abraham et al. [45] conducted a systematic review of all nonrandomized comparative studies of laparoscopic resection for colorectal cancer. This analysis included 6,438 resections. The authors found that laparoscopic resection took $27.6 \%$ (41 $\mathrm{min}$ ) longer to carry out than open resection. There was no significant difference between the 2 groups in early mortality rates or need for reoperation. The time until passage of first flatus and bowel movement, tolerating oral fluids and a solid diet was 1.2 to 1.6 days ( 26 to $37 \%$ ) shorter in the laparoscopic group; measurements of pain and narcotic analgesic requirements were 16 to $35 \%$ lower and hospital stay was 3.5 days (18.8\%) shorter following laparoscopic resection compared with open resection. Yoshida et al. [46] similarly demonstrated that laparoscopic surgery compared with open laparotomy for cholecystectomy, reduced endogenously produced opioids and cytokines. These results suggest that laparoscopic procedures may be associated with decrease in rates of POI, but other factors may be at work and more investigation is needed.

\section{Intravenous Fluids}

A review of data related to high-volume perioperative fluid therapy by Holte et al. [47] raised concerns that resulting overhydration might be detrimental in a number of respects, including impairment of cardiac and pulmonary function and the development of gut edema which would worsen POI. However, 2 more recent randomized, double-blind studies by these same authors did not validate those results, finding no increases in either ileus or hospital stay related to the volume of perioperative fluid administration [48, 49].

\section{Gastric 'Decompression' via Nasogastric Tube (NGT)} and the Use of Intraoperative Drains

Nasogastric decompression and the practice of advancing postoperative feeding only after the presence of bowel sounds and/or flatus has been a routine in surgery for decades. However, experience with laparoscopic surgery has taught us much about the archaic nature of this practice. Cheatham et al. [50] published a meta-analysis of all clinical trials comparing selective versus routine nasogastric decompression after elective laparotomy and found that routine insertion of an NGT resulted in increased adverse events including fever, pneumonia and atelectasis. They concluded that only $5 \%$ of patients stood to benefit from NGT decompression, and that it should not be routinely utilized. Whether decreasing NGT use, which is associated with decreased ambulation and in- creased patient discomfort will also decrease POI is not clear but may reasonably be expected.

Similar to the use of NGTs, an obligatory attitude toward intraoperative drain placement has been a common practice in abdominal surgery for over a century [51]. Though they effectively serve to eliminate fluid from surgical sites, they are not without risk. They are a source of wound infection and can lead to increased hospital stay [51]. Kumar et al. [51] randomized 180 patients undergoing subtotal gastrectomy into 2 groups, drain and nodrain. They found no significant difference between the groups in regard to time to return of normal GI motility, length of hospital stay, or overall mortality. Similar findings were attained by Bafna et al. [52] in patients undergoing surgery for gynecologic malignancies. Both studies concluded that drain placement is usually unnecessary since it shows no beneficial effects, except perhaps surgical peace of mind.

\section{Opioids and Opioid Antagonists}

Opioids are freely used as methods of pain control following abdominal and other surgeries. With repeated opioid administration for pain relief, tolerance to the analgesic effect subsequently develops; however, tolerance to the GI adverse effects does not $[21,53]$. Therefore, limiting opioid use postoperatively has been associated with a significant decrease in POI and remains a mainstay of POI prevention and treatment approaches $[15,54]$.

An alternative to limiting opioid use is to employ antagonists to counteract opioid effects on gastric motility. Several opiate antagonists have been found to have a beneficial effect [15]. Two novel peripheral opioid antagonists currently being investigated are alvimopan and methylnaltrexone. Both of these agents have a selective affinity for peripheral receptors and do not readily cross the blood-brain barrier. Therefore, they can reverse the negative effects of opioids on the GI tract without blocking the central opioid receptors and reversing pain relief $[15$, $22,55-57]$.

A pooled retrospective subset analysis of bowel resection found that alvimopan significantly reduced the occurrence of nausea, vomiting, NGT insertion and POI after bowel resection. Patients were given alvimopan $6 \mathrm{mg}(\mathrm{n}=397), 12 \mathrm{mg}(\mathrm{n}=413)$, or placebo $(\mathrm{n}=402)$ at least $2 \mathrm{~h}$ prior to surgery and twice daily until hospital discharge for up to 7 days. Alvimopan (6 or $12 \mathrm{mg}$ ) significantly accelerated GI recovery by 12 to $18 \mathrm{~h}$ over placebo (hazards ratio $=1.28$ and 1.38 , respectively; $\mathrm{p}<0.001$ for both). Alvimopan also significantly accelerated time to patient discharge by $16 \mathrm{~h}$ for $6 \mathrm{mg}$ and $18 \mathrm{~h}$ for $12 \mathrm{mg}$ 
( $p<0.001$ for both), from a mean of $147 \mathrm{~h}$ for placebo. In addition, alvimopan-treated patients had reduced postoperative morbidity compared with placebo, and their incidence of prolonged hospital stay or readmission was also significantly reduced $(\mathrm{p}<0.001)$ [58]. Alvimopan was generally well tolerated and the overall incidence of treatment-emergent adverse events was comparable between groups; the most commonly reported in the North American trials were nausea (alvimopan $12 \mathrm{mg}$, 55.9\%; placebo, 65.3\%) and vomiting (alvimopan $12 \mathrm{mg}, 18.8 \%$; placebo, 27.1\%) [59]. It should be noted that alvimopan is only approved for up to 15 doses since long-term treatment increased the risk for myocardial infarction within the first 4 months of therapy for patients treated with chronic opioids for pain management [60].

Methylnaltrexone is a pure $\mu$-opioid antagonist that is a quaternary derivative of naltrexone, and therefore poorly lipid soluble $[61,62]$. It does not cross the blood-brain barrier, penetrate the central nervous system, antagonize the central effects of morphine or precipitate withdrawal [61]. Early experimental studies indicated that parenterally administered methylnaltrexone is active at peripheral rather than central opioid sites [61]. Studies in healthy volunteers indicated that methylnaltrexone could reverse opioid-induced bowel inhibition without reversing analgesia $[56,61]$. A randomized controlled trial of methylnaltrexone $0.3 \mathrm{mg} / \mathrm{kg}$ administered as a 20 -min intravenous infusion every $6 \mathrm{~h}$ up to 7 days versus placebo was performed in 65 patients following open segmental colonic resection. Methylnaltrexone-treated patients recovered bowel function more quickly than placebo patients in all measures of upper and lower bowel recovery; the mean differences in most of the parameters of recovery were $>1$ day. No differences in opioid use or mean pain scores were observed and the drug was well tolerated. Adverse events were similar to placebo. This study suggests that parenteral methylnaltrexone may significantly shorten the duration of postoperative bowel dysfunction following segmental colectomy [56]. At present, methylnaltrexone is not FDA approved for the treatment of POI, but is approved for the treatment of opioid-induced constipation. Further studies are ongoing.

\section{Early Ambulation}

It has long been believed that early ambulation increases GI motility and therefore reduces POI. In an effort to discern whether ambulation hastens recovery from ileus following laparotomy, Waldhausen and Schirmer [63] carefully evaluated the effects of early ambulation on 34 consecutive patients. Seromuscular bipo- lar recording electrodes were placed on the stomach, jejunum and colon at laparotomy. These recordings did not indicate any independent benefit of early ambulation on return of normal GI electrical activity. However, despite this well-designed study, early ambulation is still important to patients in other ways, and remains a major component of most multimodal fast-track approaches to reducing POI.

\section{Postoperative Feeding}

Early feeding following intra-abdominal surgery has recently gained favor based on emerging clinical studies $[64,65]$. Jeffery et al. [64] found no difference in recovery of patients receiving a clear liquid diet, compared with those receiving a regular diet beginning with the first oral intake after surgery. However, Stewart et al. [65] found that patients in an early oral feeding group passed flatus and moved their bowels much sooner than the control group thus reducing the duration of POI and hospital stay. Though these results are mixed, there is no increase in occurrence of associated complications, leading to a growing popularity of early feeding as a potential strategy to prevent POI [22].

\section{Gum Chewing}

Gum chewing has been proposed to assist in postoperative recovery of GI function by serving as a 'sham feeding', thereby stimulating associated neuronal pathways. Although a recent meta-analysis of 5 randomized, controlled studies showed that gum chewing shortened mean time to flatus by $20 \mathrm{~h}$ and time to defecation by $29 \mathrm{~h}$, there was a nonsignificant trend toward shorter hospital stay [66].

\section{Prokinetic Agents}

Whereas animal studies have suggested that prokinetic agents might be effective treatments of POI, currently approved prokinetics primarily alter upper GI motility and generally have not been effective for treating POI [8]. Traut et al. [67] examined 39 randomized control trials that included the use of erythromycin, cholecystokinin, intravenous lidocaine, neostigmine and other prokinetic agents to evaluate their benefits postoperatively. They found that the use of most agents are not supported in the postoperative period due to their lack of effect. Intravenous lidocaine and neostigmine may be beneficial in certain situations, though further studies are needed.

The effects of stimulating laxatives on POI have been investigated. Zingg et al. [68] studied 200 patients undergoing elective colorectal resections. Patients were ran- 


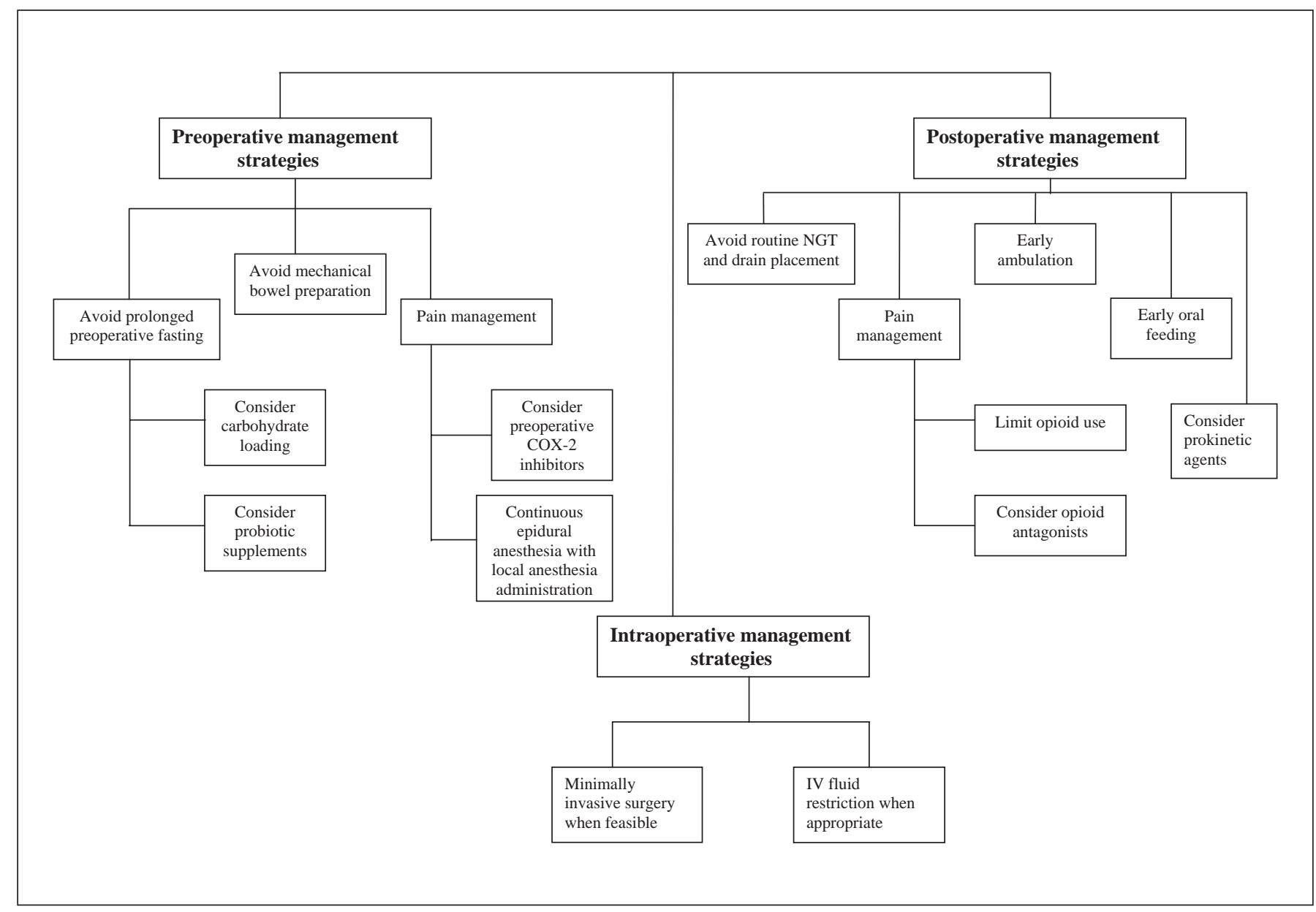

Fig. 2. Prevention and treatment algorithm for POI.

domized to receive either bisacodyl or a placebo following surgery. They found that gastrointestinal recovery was significantly accelerated in the bisacodyl group as there was a difference in time to first bowel movement by 1 day.

\section{POI: A Multifactorial Prevention and Treatment Strategy}

In the absence of any single approach that prevents or treats POI, a number of treatment algorithms have been developed which attempt to incorporate the optimal combination of the various strategies reviewed above. Although these algorithms differ in many respects, they all include limiting narcotic use and dose, incorporation of thoracic epidural analgesia with local anesthesia and emphasize early enteral feeding and ambulation (fig. 2).

Several European studies were conducted in which combinations of evidence-based perioperative strategies were instituted in a fast-track approach for patients undergoing abdominal surgery $[69,70]$. Fast-track perioperative care included extensive preoperative counseling, avoidance of mechanical bowel preparation and sedative premedication, no preoperative fasting but rather carbohydrate-loaded liquids until $2 \mathrm{~h}$ prior to surgery, tailored anesthesia encompassing thoracic epidural anesthesia and short-acting anesthetics, tight perioperative intravenous fluid restriction, minimally invasive surgical approaches when possible, nonopioid pain management, avoidance of routine drains and NGTs, standard laxatives and prokinetics, early and enhanced postoperative feeding and mobilization. In these 
studies, patients in the fast-track group achieved marked improvements in time to return of normal bowel function and reduced hospital stay compared with patients treated conventionally [71-74]. Importantly, Kehlet and Wilmore [71] point out a shared frame of mind among patients, nurses, physicians and other medical personnel are essential for the implementation of a successful fast-track protocol.

Basse et al. [75] compared 130 patients undergoing colonic surgery utilizing a fast-track protocol, with 130 patients undergoing conventional treatment. They demonstrated a reduced time to bowel movement from 4.5 to 2 days, reduced hospital stay from 8 to 4 days and reduced cardiopulmonary complications in the fast-track group. In a separate study by the same authors, 14 fasttrack patients were compared with 14 traditional patients having colonic resection. The fast-track patients reported greater reductions of time to bowel movement and length of stay than conventional patients [76]. Delaney et al. [77] have similarly demonstrated that even in more complex colorectal and reoperative pelvic surgeries fast-track treatment can significantly shorten hospital stay.

Despite the apparent effectiveness of these fast-track approaches and the negative effect of more traditional surgical practices, surgeons have been slow to adopt the new and give up the old. In a survey of clinical practice following colon operations in 295 hospitals throughout Europe and the USA, NGTs were left in situ in $40-66 \%$ of cases and preoperative mechanical bowel preps were used in more than $85 \%$ of patients [78]. Ileus persisted for more than 5 days in nearly half of these patients and hospital stay was 7-10 days. By these measures, there is still considerable progress to be made in educating today's surgeons and fully implementing steps already known to be effective in ameliorating POI.

\section{Summary}

POI is a normal consequence of abdominal and many extra-abdominal surgery. It is characterized by bowel distension, lack of bowel sounds, and a delay in time to flatus and bowel movement. Adverse effects of POI include increased postoperative pain, delay in resuming oral intake, poor wound healing, delayed mobilization, increased risk of pulmonary complications and prolonged hospitalization.

POI also has a substantial economic impact. Based on information from the HCFA database from 1999 to 2000, POI is estimated to increase hospital stay by 5 days $[1,2]$. Total hospital costs increased significantly from USD 10,000-16,000 per patient contributing to total extra hospitalization costs of USD 1.14 billion [1]. Similarly, readmission rates for patient were also found to increase from $0.2 \%$ in non-POI patients to $3.6 \%$ in those with POI $[1,2]$.

Various strategies to prevent POI and decrease the time needed for postoperative GI motility to return to normal function have been employed and/or are currently being investigated. The use of epidural local anesthetics for postoperative analgesia, when used in conjunction with fast-track perioperative care, has significantly shortened the time to return of normal bowel sounds and bowel movements. There is also clinical evidence that the use of anti-inflammatory NSAIDs and COX-2 inhibitors may help to increase GI motility after abdominal surgery. Finally, new drugs such as alvimopan and methylnaltrexone show promise for preventing POI. Though many of these strategies have been shown to be partially beneficial on there own, when used in combination as part of a fasttrack protocol a significant decrease in time to return of normal bowel function and shorten length of hospital stay can be expected.

\section{References}

Postoperative Ileus: A Review
1 Postoperative Ileus Management Council: Postoperative ileus: profiles, risk factors and definitions. Clinical consensus update in general surgery. Published 2006. http:// www.clinicalwebcasts.com/pdfs/GenSurg WEB.pdf. Accessed May 1, 2008.

2 Health Care Financing Administration: Federal Register, 1999-2000. http://www. gpoaccess.gov/fr/. Accessed May 1, 2008.

3 Iyer S, Saunders WB: Impact of postoperative ileus (POI) on hospital length of stay in colectomy surgery patients. Abstract presented at American College of Gastroenterology Annual Scientific Meeting, Philadelphia, 2007.
4 Senagore AJ: Pathogenesis and clinical and economic consequences of postoperative ileus. Am J Health Syst Pharm 2007;64:S3S7.

$\checkmark 5$ Berend KR, Lombardi AV Jr, Mallory TH, Dodds KL, Adams JB: Ileus following total hip or knee arthroplasty is associated with increased risk of deep venous thrombosis and pulmonary embolism. J Arthroplasty 2004;19:82-86.

-6 Robinson KA, Dickersin K: Development of a highly sensitive search strategy for the retrieval of reports of controlled trials using PubMed. Int J Epidemiol 2002;31:150-153. 
7 Holte K, Kehlet H: Postoperative ileus: a preventable event. Br J Surg 2000;87:14801493.

-8 Bauer AJ, Boeckxstaens GE: Mechanisms of postoperative ileus. Neurogastroenterol Motil 2004; 16:54-60.

$\checkmark 9$ Person B, Wexner SD: The management of postoperative ileus. Curr Probl Surg 2006; 43:6-65.

- 10 Bianchi C, Ballard JL, Abou-Zamzam AM, Teruya TH, Abu-Assal ML: Anterior retroperitoneal lumbosacral spine exposure: operative technique and results. Ann Vasc Surg 2003;17:137-142.

-11 Shapiro G, Green DW, Fatica NS, BoachieAdjei O: Medical complications in scoliosis surgery. Curr Opin Pediatr 2001;13:36-41.

12 Luckey A, Livingston E, Tache Y: Mechanisms and treatment of postoperative ileus. Arch Surg 2003;138:206-214.

13 Livingston EH, Passaro EP Jr: Postoperative ileus. Dig Dis Sci 1990;35:121-132.

- 14 Waldhausen JH, Shaffrey ME, Skenderis BS, Jones RS, Schirmer BD: Gastrointestinal myoelectric and clinical patterns of recovery after laparotomy. Ann Surg 1990;211:777784 .

15 Behm B, Stollman N: Postoperative ileus: etiologies and interventions. Clin Gastroenterol Hepatol 2003;1:71-80.

16 Wilson JP: Postoperative motility of the large intestine in man. Gut 1975;16:689692.

17 Woods JH, Erickson LW, Condon RE, Schulte WJ, Sillin LF: Postoperative ileus: a colonic problem? Surgery 1978;84:527-533.

18 Johnston KW: Multicenter prospective study of nonruptured abdominal aortic aneurysm. II. Variables predicting morbidity and mortality. J Vasc Surg 1989;9:437-447.

19 Artinyan A, Nunoo-Mensah JW, Balasubramaniam S, et al: Prolonged postoperative ileus: definition, risk factors, and predictors after surgery. World J Surg. DOI:10.1007/ s0026800894912.

-20 Gervaz P, Bucher P, Scheiwiller A, MugnierKonrad B, Morel P: The duration of postoperative ileus after elective colectomy is correlated to surgical specialization. Int $\mathrm{J}$ Colorectal Dis 2006;21:542-546.

21 Holte K, Kehlet H: Postoperative ileue: progress towards effective management. Drugs 2002;62:2603-2615.

-22 Clevers GJ, Smout AJ, van der Schee EJ, Akkermans LM: Myo-electrical and motor activity of the stomach in the first days after abdominal surgery: evaluation by electrogastrography and impedance gastrography. J Gastroenterol Hepatol 1991;6:253-259.

-23 Zmora O, Mahaina A, Bar-Zakai B, et al: Colon and rectal surgery without mechanical bowel preparation: a randomized prospective trial. Ann Surg 2003;237:363-367.
24 Bucher P, Mermillod B, Morel P, Soravia C: Does mechanical bowel preparation have a role in preventing postoperative complications in elective colorectal surgery? Swiss Med Wkly 2004;134:69-74.

25 Guenaga KKFG, Atallah ÁN, Castro AA, Matos D, Wille-Jørgensen P: Mechanical bowel preparation for elective colorectal surgery. Cochrane Database Syst Rev 2005; Issue 1, article No. CD001544.

26 Shafii M, Murphy DM, Donovan MG, Hickey DP: Is mechanical bowel preparation necessary in patients undergoing cystectomy and urinary diversion? BJU Int 2002;89:879881.

27 Bengmark S, Gil A: Bioecological and nutritional control of disease: prebiotics, probiotics, and synbiotics. Nutr Hosp 2006;21:7284

28 Noblett S, Watson D, Huong H, Davison B, Hainsworth P, Horgan A: Pre-operative oral carbohydrate loading in colorectal surgery: a randomized controlled trial Colorectal Surg 2006;8:563-569.

29 Nygren J, Thorell A, Ljungqvist O: Preoperative oral carbohydrate nutrition: an update. Curr Opin Clin Nutr Metab Care 2001;4 255-259.

30 Fearon KC, Luff R: The nutritional management of surgical patients: enhanced recovery after surgery. Proc Nutr Soc 2003;62:807811

31 Sim R, Cheong DM, Wong KS, Lee BM, Liew QY: Prospective randomized, double-blind, placebo-controlled study of pre- and postoperative administration of a COX-2-specific inhibitor as opioid-sparing analgesia in major colorectal surgery. Colorectal Dis 2007;9: $52-60$

32 Liu S, Carpenter RL, Neal JM: Epidural anesthesia and analgesia: their role in postoperative outcome. Anesthesiology 1995;82: 1474-1506.

33 Steinbrook RA: Epidural anesthesia and gastrointestinal motility. Anesth Analg 1998; 86:837-844.

34 Jorgensen H, Wetterslev J, Moiniche S, Dahl JB: Epidural local anaesthetics versus opioid based analgesic regimens on postoperative gastrointestinal paralysis, PONV and pain after abdominal surgery. Cochrane Database Syst Rev 2000:CD001893.

-35 Gendall KA, Kennedy RR, Watson AJ, Frizelle FA: The effect of epidural analgesia on postoperative outcome after colorectal surgery. Colorectal Dis 2007;9:584-598.

36 Marret E, Remy C, Bonnet F: Meta-analysis of epidural analgesia versus parenteral opioid analgesia after colorectal surgery. $\mathrm{Br}$ Surg 2007;94:665-673.

37 Wind J, Polle SW, Fung Kon Jin PH, et al: Systematic review of enhanced recovery programmes in colonic surgery. Br J Surg 2006; 93:800-809.
38 Buvanendran A, Kroin JS, Tuman KJ, et al: Effects of perioperative administration of a selective cyclooxygenase 2 inhibitor on pain management and recovery of function after knee replacement: a randomized controlled trial. JAMA 2003;290:2411-2418.

39 Malan TP Jr, Marsh G, Hakki SI, Grossman E, Traylor L, Hubbard RC: Parecoxib sodium, a parenteral cyclooxygenase 2 selective inhibitor, improves morphine analgesia and is opioid-sparing following total hip arthroplasty. Anesthesiology 2003;98:950-956.

40 Reynolds LW, Hoo RK, Brill RJ, North J, Recker DP, Verburg KM: The COX-2 specific inhibitor, valdecoxib, is an effective, opioid-sparing analgesic in patients undergoing total knee arthroplasty. J Pain Symptom Manage 2003;25:133-141.

-41 Lacy AM, Garcia-Valdecasas JC, Pique JM, et al: Short-term outcome analysis of a randomized study comparing laparoscopic vs. open colectomy for colon cancer. Surg Endosc 1995;9:1101-1105.

42 Schwenk W, Bohm B, Haase O, Junghans T, Muller JM: Laparoscopic versus conventional colorectal resection: a prospective randomised study of postoperative ileus and early postoperative feeding. Langenbecks Arch Surg 1998;383:49-55.

-43 Milsom JW, Bohm B, Hammerhofer KA, Fazio V, Steiger E, Elson P: A prospective, randomized trial comparing laparoscopic versus conventional techniques in colorectal cancer surgery: a preliminary report. J Am Coll Surg 1998;187:46-54.

44 Leung KL, Lai PB, Ho RL et al: Systemic cytokine response after laparoscopic-assisted resection of rectosigmoid carcinoma: a prospective randomized trial. Ann Surg 2000; 231:506-511.

45 Abraham NS, Byrne CM, Young JM, Solomon MJ: Meta-analysis of non-randomized comparative studies of the short-term outcomes of laparoscopic resection for colorectal cancer. Aust NZ J Surg 2007;77:508-516.

-46 Yoshida S, Ohta J, Yamasaki K, et al: Effect of surgical stress on endogenous morphine and cytokine levels in the plasma after laparoscopic or open cholecystectomy. Surg Endosc 2000;14:137-140.

47 Holte K, Sharrock NE, Kehlet H: Pathophysiology and clinical implications of perioperative fluid excess. Br J Anaesth 2002;89:622632.

48 Holte K, Foss NB, Andersen J, et al: Liberal or restrictive fluid administration in fasttrack colonic surgery: a randomized, doubleblind study. Br J Anaesth 2007;99:500-508.

-49 Holte K, Kristensen BB, Valentiner L, Foss NB, Husted H, Kehlet H: Liberal versus restrictive fluid management in knee arthroplasty: a randomized, double-blind study. Anesth Analg 2007;105:465-474. 
50 Cheatham ML, Chapman WC, Key SP, Sawyers JL: A meta-analysis of selective versus routine nasogastric decompression after elective laparotomy. Ann Surg 1995;221: 469-476.

-51 Kumar M, Yang S, Jaiswal V, Shah V, Shreshtha M, Gongal R: Is prophylactic placement of drains necessary after subtotal gastrectomy? World J Gastroenterol 2007; 13:3738-3741

52 Bafna U, Umadevi K, Savitha M: Closed suction drainage versus no drainage following pelvic lymphadenectomy for gynecological malignancies Int J Gynecol Cancer 2001;11: 143-146.

53 Yuan CS, Foss JF: Antagonism of gastrointestinal opioid effects. Reg Anesth Pain Med 2000;25:639-642.

54 Ferraz AA, Cowles VE, Condon RE, et al: Nonopioid analgesics shorten the duration of postoperative ileus. Am Surg 1995;61: 1079-1083.

55 Delaney CP: Clinical perspective on postoperative ileus and the effect of opiates. Neurogastroenterol Motil 2004;16:61-66.

$\checkmark 56$ Yuan CS, Israel RJ: Methylnaltrexone, a novel peripheral opioid receptor antagonist for the treatment of opioid side effects. Expert Opin Investig Drugs 2006;15:541-552.

57 Yuan CS: Methylnaltrexone mechanisms of action and effects on opioid bowel dysfunction and other opioid adverse effects. Ann Pharmacother 2007;41:984-993.

$\checkmark 58$ Delaney CP, Wolff BG, Viscusi ER, et al: Alvimopan, for postoperative ileus following bowel resection: a pooled analysis of phase III studies. Ann Surg 2007;245:355-363.

59 Senagore AJ, Bauer JJ, Du W, Techner L: Alvimopan accelerates gastrointestinal recovery after bowel resection regardless of age, gender, race, or concomitant medication use. Surgery 2007; 142:478-486.

60 Food and Drug Administration: http://www. fda.gov/cder/foi/label/2008/021775REMS. pdf

-61 Kurz A, Sessler DI: Opioid-induced bowel dysfunction: pathophysiology and potential new therapies. Drugs 2003;63:649-671.
62 Goodman AJ, Le BB, Dolle RE: Mu opioid receptor antagonists: recent developments. Chem Med Chem 2007;2:1552-1570.

63 Waldhausen JH, Schirmer BD: The effect of ambulation on recovery from postoperative ileus. Ann Surg 1990;212:671-677.

64 Jeffery KM, Harkins B, Cresci GA, Martindale RG: The clear liquid diet is no longer a necessity in the routine postoperative management of surgical patients. Am Surg 1996; 62:167-170.

65 Stewart BT, Woods RJ, Collopy BT, Fink RJ Mackay JR, Keck JO: Early feeding after elective open colorectal resections: a prospective randomized trial. Aust NZ J Surg.1998;68: 125-128.

66 de Castro SM, van den Esschert JW, van Heek NT, et al: A systematic review of the efficacy of gum chewing for the amelioration of postoperative ileus. Dig Surg 2008;25:3945

67 Traut U, Brügger L, Kunz R, Pauli-Magnus C, Haug K, Bucher H, Koller MT: Systemic prokinetic pharmacologic treatment for postoperative adynamic ileus following abdominal surgery in adults. Cochrane Database Syst Rev 2008; Issue 1, article No. CD004930.

68 Zingg U, Miskovic D, Pasternak I, Meyer P, Hamel CT, Metzger U: Effect of bisacodyl on postoperative bowel motility in elective colorectal surgery: a prospective, randomized trial. Int J Colorectal Dis 2008;23:11751183.

69 Merat S, Rouquie D, Bordier E, Legulluche Y Baranger B: Fast track rehabilitation in colonic surgery (in French). Ann Fr Anesth Reanim 2007;26:649-655

70 Mattei P, Rombeau JL: Review of the pathophysiology and management of postoperative ileus. World J Surg 2006;30:1382-1391.

71 Kehlet H, Wilmore DW: Multimodal strategies to improve surgical outcome. Am J Surg 2002;183:630-641.

72 Wind J, Hofland J, Preckel B, et al: Perioperative strategy in colonic surgery: laparoscopy and/or FAst track multimodal management versus standard care (LAFA trial). BMC Surg 2006;6:16.

73 Wilmore DW, Kehlet H: Management of patients in fast track surgery. BMJ 2001;322 473-476.

74 Kehlet H, Wilmore DW: Fast-track surgery. Br J Surg 2005;92:3-4.
75 Basse L, Thorbol JE, Lossl K, Kehlet H: Colonic surgery with accelerated rehabilitation or conventional care. Dis Colon Rectum 2004;47:271-277.

76 Basse L, Raskov HH, Hjort JD, et al: Accelerated postoperative recovery program after colonic resection improves physical performance, pulmonary function and body composition. Br J Surg 2002;89:446-453.

77 Delaney CP, Fazio VW, Senagore AJ, Robinson B, Halverson AL, Remzi FH: 'Fast track' postoperative management protocol for patients with high co-morbidity undergoing complex abdominal and pelvic colorectal surgery. Br J Surg 2001;88:1533-1538.

$>78$ Kehlet H, Buchler MW, Beart RW Jr, Billingham RP, Williamson R: Care after colonic operation - is it evidence-based? Results from a multinational survey in Europe and the United States. J Am Coll Surg 2006;202: 45-54.

79 Kalff JC, Schraut WH, Simmons RL, Bauer AJ: Surgical manipulation of the gut elicits an intestinal muscularis inflammatory response resulting in postsurgical ileus. Ann Surg 1998;228:652-663.

-80 Schwarz NT, Beer-Stolz D, Simmons RL, Bauer AJ: Pathogenesis of paralytic ileus: intestinal manipulation opens a transient pathway between the intestinal lumen and the leukocytic infiltrate of the jejunal muscularis. Ann Surg 2002;235:31-40.

81 Schwarz NT, Kalff JC, Turler A et al: Prostanoid production via COX-2 as a causative mechanism of rodent postoperative ileus. Gastroenterology 2001;121:1354-1371.

$>82$ Kalff JC, Buchholz BM, Eskandari MK, et al: Biphasic response to gut manipulation and temporal correlation of cellular infiltrates and muscle dysfunction in rat. Surgery 1999; 126:498-509.

$>83$ Bauer AJ, Sarr MG, Szurszewski JH: Opioids inhibit neuromuscular transmission in circular muscle of human and baboon jejunum. Gastroenterology 1991;101:970-976.

84 Bauer AJ, Szurszewski JH: Effect of opioid peptides on circular muscle of canine duodenum. J Physiol 1991;434:409-422. 